\title{
Analysis and Improvements of Two Identity-Based Perfect Concurrent Signature Schemes*
}

\author{
Zhenjie HUANG ${ }^{a, b}, \mathrm{Kefei} \mathrm{CHEN}^{b}$, Xuanzhi LIN $^{a}$, Rufen $\mathrm{HUANG}^{a}$ \\ a) Department of Computer Science and Engineering, Zhangzhou Normal University \\ Fujian, 363000, P. R. China \\ e-mail: zjhuang@fjzs.edu.cn \\ b) Department of Computer Science and Engineering, Shanghai Jiao Tong University \\ Shanghai 200030, P. R. China
}

Received: January 2007

\begin{abstract}
The notion of concurrent signatures was introduced by Chen, Kudla and Paterson in their seminal paper in Eurocrypt 2004. In concurrent signature schemes, two entities can produce two signatures that are not binding, until an extra piece of information (namely the keystone) is released by one of the parties. Upon release of the keystone, both signatures become binding to their true signers concurrently. In ICICS 2005, two identity-based perfect concurrent signature schemes were proposed by Chow and Susilo. In this paper, we show that these two schemes are unfair. In which the initial signer can cheat the matching signer. We present a formal definition of ID-based concurrent signatures which redress the flaw of Chow et al.'s definition and then propose two simple but significant improvements to fix our attacks.
\end{abstract}

Key words: concurrent signature, identity-based, bilinear pairings, cryptoanalysis, fair exchange.

\section{Introduction}

\subsection{Background}

The problem of fair exchange of signatures is a fundamental and well-studied problem in cryptography, with potential application in a wide range of scenarios in which the parties involved are mutually distrustful. Early work on solving the problem of fair exchange of signatures was based on the idea of timed release or timed fair exchange of signatures (Boneh and Naor, 2000; Even et al., 1985; Garay and Pomerance, 2003). Such protocols are highly interactive with many message flows and may be too interactive for many applications. Another approach to solving this problem involves the use of a trusted (or semi-trusted) third party or arbitrator who can be called upon to handle disputes between

${ }^{*}$ This work is supported by the National Natural Science Foundation of China under Grant No.60673078, the China Postdoctoral Science Foundation under Grant No. 20060390152, the Natural Science Foundation of Fujian Province of China under Grant No.2006J0045 and the Innovation Foundation for Young Technological Talents of Fujian Province of China under Grant No.2005J055. 
signers (Asokan et al., 1998; Boneh et al., 2003; Camenisch and Shoup, 2003; Garay et al., 1999; Goldreich, 1983). The main problem with such an approach is the requirement for a dispute-resolving third party with functions beyond those required of a normal Certification Authority. In general, appropriate third parties may not be available.

Concurrent signatures were introduced as an alternative approach to solving the problem of fair exchange of signatures by Chen, Kudla and Paterson (Chen et al., 2004) in their seminal paper in Eurocrypt 2004. In concurrent signature schemes, two entities can produce two signatures that are not binding, until an extra piece of information (namely the keystone) is released by one of the parties. Upon release of the keystone, both signatures become binding to their true signers concurrently. Concurrent signatures have a benefit that they have none of the disadvantages of previous fair exchange protocols: they require neither special trusted third party nor highly interactive.

The concept of identity-based (simply ID-based) public key cryptosystem was introduced in 1984 by Shamir (1985). ID-based cryptosystems (Gangishetti et al., 2006; Kancharla et al., 2007) have a property that a userÆs public key can be easily calculated from his identity by a publicly available function, while his private key can be calculated for him by a trusted authority, called Key Generation Center (KGC). They enable any pair of users to communicate securely without exchanging public key certificates, without keeping a public key directory, and without using online service of a third party, as long as a trusted key generation center issues a private key to each user when he first joins the network, so they can be a good alternative for certificate-based public key infrastructure, especially when efficient key management and moderate security are required.

\subsection{Previous Works}

In (Chen et al., 2004), Chen et al. proposed a concrete concurrent signature scheme based on a variant of Schnorr based ring signature scheme. In their scheme, before the keystone is released, any third party cannot be convinced that a signature has indeed been signed by one particular signer, since any signer can always generate this signature by himself/herself. Later, Susilo et al. pointed out that in a situation where the initial signer and the matching signer are known to be honest players (Susilo et al., 2004). In Chen et al.'s scheme, any third party can be sure that both signers have signed the messages even before the keystone is released. Then, they extended the notion of concurrent signatures to a stronger notion of perfect concurrent signatures, which will allow full ambiguity of the concurrent signatures, even both signers are known to be trustworthy. They proposed two concrete schemes to satisfy this model. The first scheme is based on a variant of Schnorr ring signature scheme, and the second scheme is based on bilinear pairing. In 2005, there are four concurrent signature schemes have been proposed. Susilo and $\mathrm{Mu}$ proposed a tripartite concurrent signature scheme from bilinear pairings (Susilo and $\mathrm{Mu}$, 2005). In tripartite concurrent signatures, three parties can exchange their signatures in such a way that their signatures will be binding concurrently. Chow and Susilo proposed two identity-based (simply ID-based) perfect concurrent signature schemes based on two major paradigms of ID-based ring signature schemes (Chow and Susilo, 2005). Previous concurrent signature schemes use the concept of ring signatures in their construction. 
Nguyen proposed a new concurrent signature (namely asymmetric concurrent signatures) scheme which is independent of the ring signature concept (Nguyen, 2005). This scheme based on Schnorr signature scheme and Schnorr-like signature scheme. Recently, Tonien et al. proposed a multi-party concurrent signature scheme using techniques of ring signatures and bilinear pairings (Tonien et al., 2006).

\subsection{Contribution}

In this paper, we show that Chow et al.'s two ID-based perfect concurrent signature schemes (Chow and Susilo, 2005) are unfair, in their schemes the initial signer Alice can cheat the matching signer Bob. We will show that. In their schemes, by carefully choosing some communication values, Alice can perform the signature protocol with Bob on messages $m_{A}$ and $m_{B}$, but outputs a valid signature pair $\left(\widetilde{\sigma}, \sigma_{B}\right)$ on $\left(\widetilde{m}, m_{B}\right)$ with $\widetilde{m} \neq m_{A}$. We give two attacks for each Chow et al.'s schemes, respectively.

For the case of one keystone only, Chen et al. presented a formal definition of fairness in (Chen et al., 2004), but it is no longer appropriate in the case of two or more keystones. Chen et al. did not present any new definition of fairness (Chow and Susilo, 2005). As a result, their schemes are unfair. Furthermore, the definition of ID-based concurrent signatures given by (Chow and Susilo, 2005) implies that two keystones $k_{I}$ and $k_{M}$ are chosen by the initial signer. As mentioned above, it may cause unfair. In this paper, we present a formal definition of ID-based concurrent signatures which redress the flaw of Chow et al.'s definition. We then propose two simple but significant improvements to fix our attacks.

\subsection{Organization}

The rest of this paper is organized as follows. The notions of Bilinear Pairings, a complexity assumption and Chow et al.'s schemes are given in Section 2. Attacks on the fairness of Chow et al.'s schemes are given in Section 3. We give a modified definition of perfect concurrent signatures in Section 4. In Section 5, we propose two improved identity-based perfect concurrent signature schemes with proofs of their securities. Section 5 concludes this paper.

\section{Review of Chow et al.'s Schemes}

\subsection{Bilinear Pairings and Complexity Assumption}

Let $\mathbb{G}_{1}$ be a cyclic additive group generated by $P$ with order prime $q$, and $\mathbb{G}_{2}$ be a cyclic multiplicative group with the same order $q$. A bilinear pairing is a map $\hat{e}: \mathbb{G}_{1} \times \mathbb{G}_{1} \rightarrow \mathbb{G}_{2}$ with the following properties:

Bilinear: For all $P, P_{1}, P_{2}, Q, Q_{1}, Q_{2} \in \mathbb{G}_{1}$,

$$
\hat{e}\left(P_{1}+P_{2}, Q\right)=\hat{e}\left(P_{1}, Q\right) \hat{e}\left(P_{2}, Q\right), \quad \hat{e}\left(P, Q_{1}+Q_{2}\right)=\hat{e}\left(P, Q_{1}\right) \hat{e}\left(P, Q_{2}\right) .
$$


Non-degenerate: There exists $P, Q \in \mathbb{G}_{1}$ such that $\hat{e}(P, Q) \neq 1$;

Computable: There is an efficient algorithm to compute $\hat{e}(P, Q)$ for all $P, Q \in \mathbb{G}_{1}$.

Modified Weil pairing and Tate pairings are examples of bilinear maps.

Computational Co-Diffie-Hellman (Co-CDH) Problem. Given a randomly chosen $\left(P_{1}, P_{2}, a P_{1}, b P_{2}\right)$, where $P_{1}, P_{2} \in \mathbb{G}_{1}, a, b \in \mathbb{Z}_{q}^{*}$, and $a, b$ are unknown, compute $a b P_{2} \in \mathbb{G}_{2}$.

Co-CDH Assumption. For every probabilistic polynomial-time algorithm $\mathcal{A}$, the advantage of $\mathcal{A}$ to solve Co-CDH-Problem is negligible.

\subsection{Chow et al.'s Scheme 1}

\subsubsection{Concurrent Signature Algorithms}

- SETUP: Choose $\left(\mathbb{G}_{1}, \mathbb{G}_{2}, \hat{e}, q, P\right)$ as subsection 2.1. The Private Key Generator (PKG) selects a random number $s \in \mathbb{Z}_{q}^{*}$ and sets $P_{p u b}=s P$. It selects three cryptographic hash functions $H_{0}:\{0,1\}^{*} \rightarrow \mathbb{G}_{1}$ and $H_{1}:\{0,1\}^{*} \rightarrow \mathbb{Z}_{q}$ and $H_{2}$ : $\{0,1\}^{*} \rightarrow \mathbb{G}_{1}$. It publishes system parameters params $=\left\{\mathbb{G}_{1}, \mathbb{G}_{2}, \hat{e}, q, P, P_{\text {pub }}\right.$, $\left.H_{0}, H_{1}, H_{2}\right\}$, and keeps $s$ as the master private key. The algorithm also sets $\mathcal{M}=\mathcal{K}_{I}=\mathcal{K}_{M}=\mathcal{F}=\mathbb{Z}_{q}$ and $\mathcal{K}^{\prime}=\mathbb{G}_{1}$.

- EXTRACT: The EXTRACT algorithm is defined as follows:

- a user $\mathcal{U}_{i}$ submits his or her identity $I D_{i}$ to the PKG;

- the PKG generates $\mathcal{U}_{i}$ 's private key as $S_{I D_{i}}=s Q_{I D_{i}}$, where $Q_{I D_{i}}=H_{0}\left(I D_{i}\right)$.

- FIX-INITIAL-KEYSTONE: Assuming a keystone $k_{I} \in \mathbb{Z}_{q}$ is randomly selected, this algorithm outputs $f_{I}=H_{1}\left(k_{I}\right)$ as the keystone fix.

- ASIGN: The ASIGN algorithm accepts the following parameters $\left(I D_{i}, I D_{j}, S_{I D_{i}}\right.$, $\alpha, f, m)$, where $S_{I D_{i}}$ is the private key associated with $Q_{I D_{i}}, \alpha, f \in \mathcal{F}$ and $m \in \mathcal{M}$. The algorithm will perform the following:

- select a random point $Z \in \mathbb{G}_{1}$;

- set $u_{j}=\alpha \cdot f$;

- compute $u_{0}=H_{1}\left(H_{2}(m)\left\|\left(I D_{i} \oplus I D_{j}\right)\right\| \hat{e}(Z, P) \hat{e}\left(u_{j} Q_{I D_{j}}, P_{\text {pub }}\right)\right)$;

- compute $V=u_{0}^{-1}\left(Z-\left(u_{0}-u_{j}\right) S_{I D_{i}}\right)$;

- output $\sigma=\left(u_{i}=u_{0}-u_{j}, u_{j}, V\right)$ as the signature on message $m$.

- ENC-MATCHING-KEYSTONE: Assuming a keystone $k_{M} \in \mathbb{Z}_{q}$ is randomly selected, this algorithm outputs $K_{M}=k_{M} P$ as the encrypted keystone.

- FIX-SECRET-KEYSTONE: This algorithm returns $f_{S}=H_{1}\left(\hat{e}\left(K_{M}, S_{I D_{j}}\right)\right)$.

- AVERIFY: The algorithm accepts $\left(\sigma, I D_{i}, I D_{j}, m\right)$, where $\sigma=\left(u_{i}, u_{j}, V\right)$, and verifies whether

$$
u_{i}+u_{j}=H_{1}\left(H_{2}(m)\left\|\left(I D_{i} \oplus I D_{j}\right)\right\| R\right),
$$

where $R=\hat{e}(V, P)^{u_{i}+u_{j}} \hat{e}\left(u_{i} Q_{I D_{i}}, P_{p u b}\right) \hat{e}\left(u_{j} Q_{I D_{j}}, P_{p u b}\right)$. If so, then output accept. Otherwise, output reject.

- VERIFY-INITIAL-KEYSTONE: This algorithm outputs accept if $f_{I}=H_{1}\left(k_{I}\right)$, reject otherwise. 
- VERIFY-SECRET-KEYSTONE: It outputs accept if $f_{S}=H_{1}\left(\hat{e}\left(P_{p u b}, Q_{I D_{j}}\right)^{k_{M}}\right)$, reject otherwise.

- VERIFY-CONNECTION: This algorithm outputs accept if $u_{j}=f_{I}$ and $u_{i}^{\prime}=u_{j} \cdot f_{S}$, reject otherwise.

- VERIFY: The algorithm accepts $\left(k_{I}, k_{M}, S^{\prime}\right)$, where $k_{I} \in \mathcal{K}_{I}$ and $k_{M} \in \mathcal{K}_{M}$ are the keystones and $S^{\prime}=\left(\sigma_{i}, \sigma_{j}, I D_{i}, I D_{j}, m_{i}, m_{j}\right)$. The algorithm verifies whether $\left(k_{I}, k_{M}\right)$ is valid and the connection between $\sigma_{i}$ and $\sigma_{j}$ is valid by using the above three algorithms. If it does not hold, then output reject. Otherwise, run $\operatorname{AVERIFY}(S)$. The output of VERIFY is the output of AVERIFY algorithm.

2.2.2. Concurrent Signature Protocol

- Alice performs the following:

- picks a random keystone $\left(k_{I}, k_{M}\right) \in \mathbb{Z}_{q} \times \mathbb{Z}_{q}$;

- computes keystone fix $f_{I}=H_{1}\left(k_{I}\right)$;

- selects a message $m_{A} \in \mathcal{M}$, computes her ambiguous signature as $\sigma_{A}=$ $\left(u_{A}, u_{B}, V\right) \leftarrow \operatorname{ASIGN}\left(I D_{A}, I D_{B}, S_{I D_{A}}, 1, f_{I}, m_{A}\right)$;

- computes encrypted keystone $K_{M}=k_{M} P$;

- sends $\sigma_{A}$ and $K_{M}$ to Bob.

- Bob performs the following:

- verifies the signature $\sigma_{A}$ by testing whether $\operatorname{AVERIFY}\left(\sigma_{A}, I D_{A}, I D_{B}, m_{A}\right)=$ accept. Aborts if the equation does not hold.

- computes secret matching keystone fix $f_{S}=H_{1}\left(\hat{e}\left(K_{M}, S_{I D_{B}}\right)\right)$;

- selects a message $m_{B} \in \mathcal{M}$, and computes his ambiguous signature as $\sigma_{B}=$ $\left(u_{B}^{\prime}, u_{A}^{\prime}, V^{\prime}\right) \leftarrow \operatorname{ASIGN}\left(I D_{B}, I D_{A}, S_{I D_{B}}, u_{B}, f_{S}, m_{B}\right) ;$

- sends $\sigma_{B}$ and $f_{S}$ to Alice.

- Alice verifies $\sigma_{B}$ by testing whether $f_{S}=H_{1}\left(\hat{e}\left(P_{p u b}, Q_{I D_{B}}\right)^{k_{M}}\right), u_{A}^{\prime}=u_{B} \cdot f_{S}$, and $\operatorname{AVERIFY}\left(\sigma_{B}, I D_{B}, I D_{A}, m_{B}\right)=$ accept are held. If not, then Alice aborts. Otherwise, Alice releases the keystone $\left(k_{I}, k_{M}\right)$ to Bob and both signatures are binding concurrently.

\subsection{Chow et al.'s Scheme 2}

\subsubsection{Concurrent Signature Algorithms}

- SETUP: Basically it is the same as Scheme 1, but the description of spaces becomes $\mathcal{M}=\mathcal{K}_{I}=\mathcal{K}_{M}=\mathbb{Z}_{q}, \mathcal{F}=\mathcal{K}^{\prime}=\mathbb{G}_{1}$.

- EXTRACT: The same as Scheme 1.

- FIX-INITIAL-KEYSTONE: Assuming a keystone $k_{I} \in \mathbb{Z}_{q}$ is randomly selected, this algorithm outputs $f_{I}=H_{2}\left(k_{I}\right)$ as the keystone fix.

- ASIGN: The input of this algorithm includes two identities $I D_{i}$ and $I D_{j}$, a private key $S_{I D_{i}}$, a message $m$ and $\alpha, f \in \mathbb{G}_{1}$.

- Compute $U_{j}=\alpha+f$ and $h_{j}=H_{1}\left(m\left\|\left(I D_{i} \oplus I D_{j}\right)\right\| U_{j}\right)$.

- Choose $r_{i}^{\prime} \in \mathbb{Z}_{q}^{*}$ randomly, compute $U_{i}=r_{i}^{\prime} Q_{I D_{i}}-U_{j}-h_{j} Q_{I D_{j}}$.

- Compute $h_{i}=H_{1}\left(m\left\|\left(I D_{i} \oplus I D_{j}\right)\right\| U_{i}\right)$ and $V=\left(h_{i}+r_{i}^{\prime}\right) S_{I D_{i}}$. 
- Output the signature $\sigma=\left(U_{i}, U_{j}, V\right)$.

- ENC-MATCHING-KEYSTONE: The same as Scheme 1.

- FIX-SECRET-KEYSTONE: This algorithm returns $f_{S}=H_{2}\left(\hat{e}\left(K_{M}, S_{I D_{j}}\right)\right)$.

- AVERIFY: The input of this algorithm includes two identities $I D_{i}$ and $I D_{j}$, a message $m$, and a ring signature $\sigma=\left(U_{i}, U_{j}, V\right)$.

- Compute $h_{i}=H_{1}\left(m \|\left(I D_{i} \oplus I D_{j}\right)|| U_{i}\right)$ and $h_{j}=H_{1}\left(m\left\|\left(I D_{i} \oplus I D_{j}\right)\right\| U_{j}\right)$.

- Return accept if $\hat{e}\left(P_{p u b}, U_{i}+h_{i} Q_{I D_{i}}+U_{j}+h_{j} Q_{I D_{j}}\right)=\hat{e}(P, V)$, reject otherwise.

- VERIFY-INITIAL-KEYSTONE: This algorithm outputs accept if $f_{I}=H_{2}\left(k_{I}\right)$, reject otherwise.

- VERIFY-SECRET-KEYSTONE: It outputs accept if $f_{S}=H_{2}\left(\hat{e}\left(P_{p u b}, Q_{I D_{j}}\right)^{k_{M}}\right)$, reject otherwise.

- VERIFY-CONNECTION: This algorithm outputs accept if $U_{j}=f_{I}$ and $U_{i}^{\prime}=U_{j}+$ $f_{S}$, reject otherwise.

- VERIFY: The algorithm accepts $\left(k_{I}, k_{M}, S^{\prime}\right)$, where $k_{I} \in \mathcal{K}_{I}$ and $k_{M} \in \mathcal{K}_{M}$ are the keystones and $S^{\prime}=\left(\sigma_{i}, \sigma_{j}, I D_{i}, I D_{j}, m_{i}, m_{j}\right)$. The algorithm verifies whether $\left(k_{I}, k_{M}\right)$ is valid and the connection between $\sigma_{i}$ and $\sigma_{j}$ is valid by using the above three algorithms. If it does not hold, then output reject. Otherwise, run AVERIFY $(S)$. The output of VERIFY is the output of AVERIFY algorithm.

\subsubsection{Concurrent Signature Protocol}

Alice performs the following:

- picks a random keystone $\left(k_{I}, k_{M}\right) \in \mathcal{K}_{I} \times \mathcal{K}_{M}$;

- computes keystone fix $f_{I}=H_{2}\left(k_{I}\right)$;

- selects a message $m_{A} \in \mathcal{M}$, computes her ambiguous signature as $\sigma_{A}=$ $\left(U_{A}, U_{B}, V\right) \leftarrow \operatorname{ASIGN}\left(I D_{A}, I D_{B}, S_{I D_{A}}, \mathcal{O}_{\mathcal{F}}, f_{I}, m_{A}\right)$, where $\mathcal{O}_{\mathcal{F}}$ denotes the identity element of the group $\mathcal{F}$;

- computes encrypted keystone $K_{M}=k_{M} P$;

- sends $\sigma_{A}$ and $K_{M}$ to Bob.

- Bob performs the following:

- verifies the signature $\sigma_{A}$ by testing whether $\operatorname{AVERIFY}\left(\sigma_{A}, I D_{A}, I D_{B}, m_{A}\right) \stackrel{?}{=}$ accept holds; aborts if the equation does not hold;

- computes secret matching keystone fix $f_{S}=H_{2}\left(\hat{e}\left(K_{M}, S_{I D_{B}}\right)\right)$;

- selects a message $m_{B} \in \mathcal{M}$, and computes his ambiguous signature as $\sigma_{B}=$ $\left(U_{B}^{\prime}, U_{A}^{\prime}, V^{\prime}\right) \leftarrow \operatorname{ASIGN}\left(I D_{B}, I D_{A}, S_{I D_{B}}, U_{B}, f_{S}, m_{B}\right) ;$

- sends $\sigma_{B}$ and $f_{S}$ to Alice.

- Alice verifies $\sigma_{B}$ by testing whether $f_{S}=H_{2}\left(\hat{e}\left(P_{p u b}, Q_{I D_{B}}\right)^{k_{M}}\right), U_{A}^{\prime}=U_{B}+f_{S}$ and $\operatorname{AVERIFY}\left(\sigma_{B}, I D_{B}, I D_{A}, m_{B}\right)=$ accept are held. If not, then Alice aborts. Otherwise, Alice releases the keystone $\left(k_{I}, k_{M}\right)$ to Bob and both signatures are binding concurrently. 


\section{Attacks on the Fairness of Chow et al.'s Schemes}

In Chow et al.'s schemes, both keystones $k_{I}$ and $k_{M}$ are chosen by the initial signer Alice, so she can cheat the matching signer Bob by carefully choosing some communication values. We will show that Alice can perform the signature protocol with Bob on messages $m_{A}$ and $m_{B}$, but outputs a valid signature pair $\left(\widetilde{\sigma}, \sigma_{B}\right)$ on $\left(\widetilde{m}, m_{B}\right)$ with $\widetilde{m} \neq m_{A}$. Bob can still obtain a signature pair $\left(\sigma_{A}, \sigma_{B}\right)$ on $\left(m_{A}, m_{B}\right)$, but $\left(\sigma_{A}, \sigma_{B}\right)$ can not be accepted by verifying algorithm VERIFY.

\subsection{Attacks against Chow et al.'s Scheme 1}

\subsubsection{Attack 1 against Chow et al.'s Scheme 1}

In Chow et al.'s Scheme 1, if Alice let $f_{I}$ be equal to $H_{1}\left(k_{I}\right) f_{S}^{\prime} f^{-1}$ instead of $H_{1}\left(k_{I}\right)$ and let $K_{M}=k^{\prime} P$, where $f_{S}^{\prime}=H_{1}\left(\hat{e}\left(P_{p u b}, Q_{I D_{B}}\right)^{k_{M}}\right), f=H_{1}\left(\hat{e}\left(P_{p u b}, Q_{I D_{B}}\right)^{k^{\prime}}\right)$, then Bob will send a signature $\sigma_{B}=\left(u_{B}^{\prime}, u_{A}^{\prime}, V^{\prime}\right)$ with $u_{A}^{\prime}=H_{1}\left(k_{I}\right) f_{S}^{\prime}$ back to Alice. Then Alice can generate a new signature $\widetilde{\sigma}=\left(\widetilde{u}_{A}, \widetilde{u}_{B}, \widetilde{V}\right)$ on a new message $\widetilde{m}$ with $\widetilde{u}_{B}=H_{1}\left(k_{I}\right)$. The result is: the signature pair $\left(\widetilde{\sigma}, \sigma_{B}\right)$ with keystone $\left(k_{I}, k_{M}\right)$ should be accepted by VERIFY while the signature pair $\left(\sigma_{A}, \sigma_{B}\right)$ with keystone $\left(k_{I}, k_{M}\right)$ should be rejected by VERIFY since the outputs of VERIFY-INITIAL-KEYSTONE and VERIFYCONNECTION are "reject". Following is the detail.

- Alice performs the following:

- picks three random keystones $k_{I}, k_{M}, k^{\prime} \in \mathbb{Z}_{q}$;

- computes $f_{S}^{\prime}=H_{1}\left(\hat{e}\left(P_{p u b}, Q_{I D_{B}}\right)^{k_{M}}\right), f=H_{1}\left(\hat{e}\left(P_{p u b}, Q_{I D_{B}}\right)^{k^{\prime}}\right)$;

- computes keystone fix $f_{I}=H_{1}\left(k_{I}\right) f_{S}^{\prime} f^{-1}$;

- selects a message $m_{A} \in \mathcal{M}$, computes her ambiguous signature as $\sigma_{A}=$ $\left(u_{A}, u_{B}, V\right) \leftarrow \operatorname{ASIGN}\left(I D_{A}, I D_{B}, S_{I D_{A}}, 1, f_{I}, m_{A}\right) ;$

- computes encrypted keystone $K_{M}=k^{\prime} P$;

- sends $\sigma_{A}$ and $K_{M}$ to Bob.

- Bob performs the same as that of the original scheme. Note that, in this case, the secret matching keystone fix $f_{S}=H_{1}\left(\hat{e}\left(K_{M}, S_{I D_{B}}\right)\right)=f$, so Bob will return a signature $\sigma_{B}=\left(u_{B}^{\prime}, u_{A}^{\prime}, V^{\prime}\right)$ with $u_{A}^{\prime}=H_{1}\left(k_{I}\right) f_{S}^{\prime}$.

- Alice verifies Bob's ambiguous signature $\sigma_{B}$. If it is invalid, then she aborts. Otherwise, she performs following attack:

- computes keystone fix $\tilde{f}=H_{1}\left(k_{I}\right)$;

- selects a new message $\widetilde{m} \in \mathcal{M}$, computes her ambiguous signature as $\widetilde{\sigma}=$ $\left(\widetilde{u}_{A}, \widetilde{u}_{B}, \widetilde{V}\right) \leftarrow \operatorname{ASIGN}\left(I D_{A}, I D_{B}, S_{I D_{A}}, 1, \widetilde{f}, \widetilde{m}\right)$;

- releases the keystone $\left(k_{I}, k_{M}\right)$ publicly, and both signatures $\widetilde{\sigma}$ and $\sigma_{B}$ are binding concurrently.

REMARK. By releasing $k_{I}, k_{M}, K=\hat{e}\left(K_{M}, S_{I D_{B}}\right)$ such that $f_{I}=H_{1}\left(k_{I}\right)$ $H_{1}\left(\hat{e}\left(P_{p u b}, Q_{I D_{B}}\right)^{k_{M}}\right) H_{1}(K)^{-1}$, Bob can prove that the signature $\sigma_{A}$ was indeed issued by Alice, but he can not make $\sigma_{A}$ accepted by VERIFY. According to the definition, $\sigma_{A}$ is invalid. We would like to point out that the attack above is harmful. Getting a valid signature pair $\left(\widetilde{\sigma}, \sigma_{B}\right)$, one may not have his wits about the potential cheat. 


\subsubsection{Attack 2 against Chow et al. 's Scheme 1}

We can also implement an attack against Scheme 1 by letting $f_{I}=H_{1}\left(k_{I}\right)$ $H_{1}\left(\hat{e}\left(P_{p u b}, Q_{I D_{B}}\right)^{k_{M}}\right)^{-1}$. In this case, Bob will send a signature $\sigma_{B}=\left(u_{B}^{\prime}, u_{A}^{\prime}, V^{\prime}\right)$ with $u_{A}^{\prime}=H_{1}\left(k_{I}\right)$ back to Alice. In the end, Alice produces a new signature $\widetilde{\sigma}=$ $\left(\widetilde{u}_{A}, \widetilde{u}_{B}, \widetilde{V}\right)$ on a new message $\widetilde{m}$ with $\widetilde{u}_{B}=H_{1}\left(k_{I}\right) H_{1}\left(\hat{e}\left(P_{p u b}, Q_{I D_{A}}\right)^{\tilde{k}}\right)$. The signature pair $\left(\sigma_{B}, \widetilde{\sigma}\right)$ with keystone $\left(k_{I}, \widetilde{k}\right)$ should be accepted by VERIFY. Here Bob is the initial signer while Alice is the matching signer. Whereas the signature pair $\left(\sigma_{A}, \sigma_{B}\right)$ with keystone $\left(k_{I}, \widetilde{k}\right)$ should be rejected by VERIFY. The detail is as follows.

- Alice performs the following:

- picks a random keystone $\left(k_{I}, k_{M}\right) \in \mathbb{Z}_{q} \times \mathbb{Z}_{q}$;

- computes $f=H_{1}\left(\hat{e}\left(P_{p u b}, Q_{I D_{B}}\right)^{k_{M}}\right)$ and keystone fix $f_{I}=H_{1}\left(k_{I}\right) f^{-1}$;

- selects a message $m_{A} \in \mathcal{M}$, computes her ambiguous signature as $\sigma_{A}=$ $\left(u_{A}, u_{B}, V\right) \leftarrow \operatorname{ASIGN}\left(I D_{A}, I D_{B}, S_{I D_{A}}, 1, f_{I}, m_{A}\right)$;

- computes encrypted keystone $K_{M}=k_{M} P$;

- sends $\sigma_{A}$ and $K_{M}$ to Bob.

- Bob performs the same as that of the original scheme. Note that, in this case, Bob will return a signature $\sigma_{B}=\left(u_{B}^{\prime}, u_{A}^{\prime}, V^{\prime}\right)$ with $u_{A}^{\prime}=H_{1}\left(k_{I}\right)$.

- Alice verifies Bob's ambiguous signature $\sigma_{B}$. If it is invalid, then she aborts. Otherwise, she performs following attack:

- picks a new random keystone $\widetilde{k} \in \mathbb{Z}_{q}$;

- computes keystone fix $\widetilde{f}=H_{1}\left(\hat{e}\left(P_{p u b}, Q_{I D_{A}}\right)^{\tilde{k}}\right)$;

- selects a new message $\widetilde{m} \in \mathcal{M}$, computes her ambiguous signature as $\widetilde{\sigma}=$ $\left(\widetilde{u}_{A}, \widetilde{u}_{B}, \widetilde{V}\right) \leftarrow \operatorname{ASIGN}\left(I D_{A}, I D_{B}, S_{I D_{A}}, u_{A}^{\prime}, \widetilde{f}, \widetilde{m}\right) ;$

- releases the keystone $\left(k_{I}, \widetilde{k}\right)$ publicly, and both signatures $\sigma_{B}$ and $\widetilde{\sigma}$ are binding concurrently.

\subsection{Attacks against Chow et al.'s Scheme 2}

\subsubsection{Attack 1 against Chow et al.'s Scheme 2}

Similar to the Attack 1 against Scheme 1, we can also implement a attack against Scheme 2. In this case, we let $f_{I}$ be equal to $H_{2}\left(k_{I}\right)+f_{S}^{\prime}-f$ instead of $H_{2}\left(k_{I}\right)$ and let $K_{M}=k^{\prime} P$, where $f_{S}^{\prime}=H_{2}\left(\hat{e}\left(P_{p u b}, Q_{I D_{B}}\right)^{k_{M}}\right), f=H_{2}\left(\hat{e}\left(P_{p u b}, Q_{I D_{B}}\right)^{k^{\prime}}\right)$. Then Bob will send a signature $\sigma_{B}=\left(U_{B}^{\prime}, U_{A}^{\prime}, V^{\prime}\right)$ with $U_{A}^{\prime}=H_{2}\left(k_{I}\right)+f_{S}^{\prime}$ back to Alice. Finally, Alice generates a new signature $\widetilde{\sigma}=\left(\widetilde{U}_{A}, \widetilde{U}_{B}, \widetilde{V}\right)$ on a new message $\widetilde{m}$ with $\widetilde{U}_{B}=H_{1}\left(k_{I}\right)$. One can see that the signature pair $\left(\widetilde{\sigma}, \sigma_{B}\right)$ with keystone $\left(k_{I}, k_{M}\right)$ should be accepted by VERIFY while the signature pair $\left(\sigma_{A}, \sigma_{B}\right)$ with keystone $\left(k_{I}, k_{M}\right)$ should be rejected by VERIFY. The detail is similar to that of Attack 1 of Scheme 1. Due to the space limitation, we omit it here.

\subsubsection{Attack 2 against Chow et al.'s Scheme 2}

Similar to the Attack 2 against Scheme 1 , let $f_{I}=H_{2}\left(k_{I}\right)-H_{2}\left(\hat{e}\left(P_{p u b}, Q_{I D_{B}}\right)^{k_{M}}\right)$. Then Bob will send a signature $\sigma_{B}=\left(U_{B}^{\prime}, U_{A}^{\prime}, V_{\widetilde{U}}^{\prime}\right)$ with $\widetilde{\sim}_{A}^{\prime}=H_{2}\left(k_{I}\right)$ back to Alice. In the end, Alice produces a new signature $\widetilde{\sigma}=\left(\widetilde{U}_{A}, \widetilde{U}_{B}, \widetilde{V}\right)$ on a new message $\widetilde{m}$ with 
$\widetilde{U}_{B}=H_{1}\left(k_{I}\right) H_{1}\left(\hat{e}\left(P_{p u b}, Q_{I D_{A}}\right)^{\tilde{k}}\right)$. The signature pair $\left(\sigma_{B}, \widetilde{\sigma}\right)$ with keystone $\left(k_{I}, \widetilde{k}\right)$ is valid while the signature pair $\left(\sigma_{A}, \sigma_{B}\right)$ with keystone $\left(k_{I}, \widetilde{k}\right)$ is invalid. We omit the detail due to the same reason.

\section{Definition of Perfect ID-Based Concurrent Signatures}

The definition of ID-based concurrent signatures given by (Chow and Susilo, 2005) implies that two keystones $k_{I}$ and $k_{M}$ are chosen by the initial signer. As we have shown above, it may cause unfair. In this section, we present a modified definition of ID-based concurrent signatures which redress the flaw of Chow et al.'s definition.

\subsection{Concurrent ID-Based Signature Algorithm}

A concurrent signature protocol involves two parties Alice and Bob. Since one party needs to create the keystone fix and send the first ambiguous signature, we call this party the initial signer. The party who responds to this initial signature by creating another ambiguous signature we call the matching signer.

A perfect ID-based concurrent signature scheme is a digital signature scheme that consists of the following algorithms:

- SETUP: A probabilistic algorithm that on input a security parameter $l$, outputs the system parameters params which is the descriptions of the message space $\mathcal{M}$, the signature space $\mathcal{S}$, the private key space $\mathcal{K}_{s k}$, the keystone-pair space $\mathcal{K}_{I} \times \mathcal{K}_{M}$, the keystone fix space $\mathcal{F}$, the encrypted keystone space $\mathcal{K}^{\prime}$ and any additional system parameters $\pi$. The algorithm also outputs an initial-keystone-fix function $F_{I}$ : $\mathcal{K}_{I} \rightarrow \mathcal{F}$, a matching-keystone-fix function $F_{M}: \mathcal{K}_{M} \times \mathcal{F} \rightarrow \mathcal{F}$, a keystone encryption function Enc: $\mathcal{K}_{M} \rightarrow \mathcal{K}^{\prime}$ and a keystone decryption function Dec: $\mathcal{K}^{\prime} \times \mathcal{K}_{s k} \rightarrow \mathcal{K}_{M}$. (Note that we do not include params explicitly as the input in the following descriptions.)

- EXTRACT: A probabilistic algorithm that on inputs a participant's identity $I D$, outputs a public key $Q_{I D}$ and the corresponding private key $S_{I D}$.

- ASIGN: A probabilistic algorithm that on inputs $\left(I D_{i}, I D_{j}, S_{I D_{i}}, f, m\right)$, where $f \in$ $\mathcal{F}, I D_{i}, I D_{j}$ are the identities of the participants, $S_{I D_{i}}$ is the private key associated with $I D_{i}$ and $m \in \mathcal{M}$, outputs an ambiguous signature $\sigma=\left(u_{i}, u_{j}, V\right)$ on $m$.

- AVERIFY: A deterministic algorithm that takes as input $S=\left(\sigma, I D_{i}, I D_{j}, m\right)$ and outputs accept or reject.

- VERIFY: A deterministic algorithm that takes as input $\left(k_{I}, k_{M}, S^{\prime}\right)$, where $\left(k_{I}, k_{M}\right) \in \mathcal{K}_{I} \times \mathcal{K}_{M}, S^{\prime}=\left(\sigma_{I}, \sigma_{M}, I D_{I}, I D_{M}, m_{I}, m_{M}\right)$, and outputs accept or reject.

\subsection{Concurrent Signature Protocol}

The concurrent signature protocol works as follows.

- The initial signer performs the following: 
- picks a random keystone $k_{I} \in \mathcal{K}_{I}$, and computes the corresponding keystone fix $f_{I}=F_{I}\left(k_{I}\right)$;

- picks a message $m_{I} \in \mathcal{M}$ and computes her ambiguous signature $\sigma_{I}=$ $\left(u_{I}, u_{M}, V\right)=\operatorname{ASIGN}\left(I D_{I}, I D_{M}, S_{I D_{I}}, f_{I}, m_{I}\right)$;

- sends $\sigma_{I}$ to the matching signer.

- The matching signer performs the following:

- verifies $\sigma_{I}$ by checking whether $\operatorname{AVERIFY}\left(\sigma_{I}, I D_{I}, I D_{M}, m_{I}\right)=$ accept; if not, he aborts.

- picks a random keystone $k_{M} \in \mathcal{K}_{M}$, and computes the corresponding keystone fix $f_{M}=F_{M}\left(k_{M}, u_{M}\right)$;

- picks a message $m_{M} \in \mathcal{M}$ and computes his ambiguous signature $\sigma_{M}=$ $\left(u_{M}^{\prime}, u_{I}^{\prime}, V^{\prime}\right)=\operatorname{ASIGN}\left(I D_{M}, I D_{I}, S_{I D_{M}}, f_{M}, m_{M}\right)$;

- computes the encrypted keystone $K_{M}=\operatorname{Enc}\left(k_{M}\right)$;

- sends $\sigma_{M}$ and $K_{M}$ back to the initial signer.

- The initial signer performs the following:

- computes the matching keystone $k_{M}=\operatorname{Dec}\left(K_{M}, S_{I D_{I}}\right)$;

- verifies $\sigma_{M}$ by checking whether AVERIFY $\left(\sigma_{M}, I D_{M}, I D_{I}, m_{M}\right)=$ accept and $u_{I}^{\prime}=F_{M}\left(k_{M}, f_{I}\right)$ are held. If not, she aborts. Otherwise, she releases the keystone pair $\left(k_{I}, k_{M}\right)$.

\subsection{Security Model for Perfect Concurrent Signatures}

A secure perfect concurrent signature scheme should have five properties: correctness, unforgeability, ambiguity, unlinkability and fairness.

Correctness. If a signature $\sigma$ has been generated correctly by invoking ASIGN algorithm on a message $m \in \mathcal{M}$, AVERIFY algorithm will return accept with an overwhelming probability, given a signature $\sigma$ on $m$ and a security parameter $l$. After the keystone pair $\left(k_{I}, k_{M}\right) \in \mathcal{K}_{I} \times \mathcal{K}_{M}$ is released, the output of VERIFY algorithm will be accept with an overwhelming probability.

Unforgeability. Unforgeability for a concurrent signature under a chosen message attack is defined by the following game between an adversary $\mathcal{A}$ and a challenger $\mathcal{C}$.

- Setup: $\mathcal{C}$ runs SETUP for a given security parameter $l$ to obtain the system parameters params.

- Queries: $\mathcal{A}$ can make the following types of query to the challenger $\mathcal{C}$ :

- Hash Function Query: $\mathcal{A}$ can request a value of Hash function for any input. $\mathcal{C}$ computes and outputs the value of the Hash function for the requested input.

- EXTRACT Query: $\mathcal{A}$ can request a private key for any input $I D . \mathcal{C}$ runs EXTRACT and outputs the corresponding private key $S_{I D}$.

- $F_{I}$ Query: $\mathcal{A}$ can request that $\mathcal{C}$ select a keystone $k_{I}$ and return the keystone fix $f_{I}=F_{I}\left(k_{I}\right)$. If $\mathcal{A}$ wishes to choose his own keystone $k_{I}$, then he can request the keystone fix $f_{I}=F_{I}\left(k_{I}\right)$ by a $F_{I}$ Query with input $k_{I}$.

- $F_{M}$ Query: $\mathcal{A}$ can request a matching keystone fix corresponding to a keystone fix $f \in \mathcal{F}$. $\mathcal{C}$ selects a keystone $k_{M}$ and returns the keystone fix 
$f_{M}=F_{M}\left(k_{M}, f\right)$. If $\mathcal{A}$ wishes to choose his own keystone $k_{M}$, then he can request the keystone fix $f_{M}=F_{M}\left(k_{M}, f\right)$ by a $F_{M}$ Query with input $\left(k_{M}, f\right)$.

- $F_{I}$ Reveal Query: $\mathcal{A}$ can request that $\mathcal{C}$ reveal the keystone $k_{I}$ that he used to produce a keystone fix $f_{I} \in \mathcal{F}$ in a previous $F_{I}$ query. If $f_{I}$ was not a previous $F_{I}$ output, then $\mathcal{C}$ outputs invalid. Otherwise, $\mathcal{C}$ outputs $k_{I}$ where $f_{I}=F_{I}\left(k_{I}\right)$.

- $F_{M}$ Reveal Query: $\mathcal{A}$ can request that $\mathcal{C}$ reveal the keystone $k_{M}$ and the keystone fix $f$ that he used to produce a keystone fix $f_{M} \in \mathcal{F}$ in a previous $F_{M}$ query. If $f_{M}$ was not a previous $F_{M}$ output, then $\mathcal{C}$ outputs invalid. Otherwise, $\mathcal{C}$ outputs $k_{M}$ and $f$ such that $f_{M}=F_{I}\left(k_{M}, f\right)$.

- ASIGN Query: $\mathcal{A}$ can request an ambiguous signature for any input of the form $\left(I D_{i}, I D_{j}, f_{i}, m_{i}\right)$ where $f_{i} \in \mathcal{F}, I D_{i}, I D_{j} \neq I D_{i}$ are the identities of the participants and $m_{i} \in \mathcal{M}$. $\mathcal{C}$ responds with an ambiguous signature $\sigma=\left(u_{i}, u_{j}, V\right)$.

- Output: Finally, $\mathcal{A}$ outputs a tuple $\sigma=\left(u_{c}, u_{d}, V\right)$ where $u_{c}, u_{d} \in \mathcal{F}$, along with identities $I D_{c}$ and $I D_{d}$, and a message $m \in \mathcal{M}$.

The adversary wins the game if $\operatorname{AVERIFY}\left(\sigma, I D_{c}, I D_{d}, m\right)=$ accept , and if either of the following two cases hold:

- No ASIGN query with input either of the tuples $\left(I D_{c}, I D_{d}, u_{d}, m\right)$ or $\left(I D_{d}, I D_{c}, u_{c}, m\right)$ was made by $\mathcal{A}$ and no EXTRACT query was made by $\mathcal{A}$ on either $I D_{c}$ or $I D_{d}$.

- No ASIGN query with input of the tuples $\left(I D_{c}, I D_{i}, u_{d}, m\right)$ was made by $\mathcal{A}$ for any $I D_{i} \neq I D_{c}$, no EXTRACT query was made by $\mathcal{A}$ on $I D_{c}$ and either $u_{d}$ was a previous output from a $F_{I}$ ( or $F_{M}$ ) query or $\mathcal{A}$ produces a keystone $k$ such that $u_{d}=F_{I}(k)\left(\right.$ or $\left(k, k^{\prime}\right)$ such that $\left.u_{d}=F_{M}\left(k, F_{I}\left(k^{\prime}\right)\right)\right)$.

DEFINITION 1. We say that an ID-based concurrent signature scheme is existentially unforgeable under a chosen message attack if the probability of success of any polynomially bounded adversary in the above game is negligible.

Ambiguity. Ambiguity for a concurrent signature scheme is defined by the following game between an adversary $\mathcal{A}$ and a challenger $\mathcal{C}$.

- Setup: This is as above in the unforgeability game.

- Phase 1: $\mathcal{A}$ makes a sequence of EXTRACT, $F_{I}, F_{M}, F_{I}$ Reveal, $F_{M}$ Reveal, ASIGN queries. These queries are answered by $\mathcal{C}$ as in the above unforgeability game.

- Challenge: $\mathcal{A}$ selects a challenge tuple $\left(I D_{i}, I D_{j}, m\right)$ where $I D_{i}, I D_{j}$ are the identities of the participants and $m \in \mathcal{M}$. In response, $\mathcal{C}$ randomly selects $k \in \mathcal{K}_{I}$ and computes $f=F_{I}(k)$ or randomly selects $\left(k, k^{\prime}\right) \in \mathcal{K}_{M} \times \mathcal{K}_{I}$ and computes $f=F_{M}\left(k, F_{I}\left(k^{\prime}\right)\right)$ (each with probability of $1 / 2$ ), then randomly selects a bit $b \in\{0,1\}$. $\mathcal{C}$ outputs $\sigma=\operatorname{ASIGN}\left(I D_{i}, I D_{j}, S_{I D_{i}}, f, m\right)$ if $b=0$; otherwise $\mathcal{C}$ outputs $\sigma=\operatorname{ASIGN}\left(I D_{j}, I D_{i}, S_{I D_{j}}, f, m\right)$. Denoted the outputed signature $\sigma$ by $\left(u_{1}, u_{2}, V\right)$. 
- Phase 2: $\mathcal{A}$ may make another sequence of queries as in Phase 1; these are handled by $\mathcal{C}$ as before.

- Output: Finally, $\mathcal{A}$ outputs a guess bit $b^{\prime} \in\{0,1\}$. $\mathcal{A}$ wins if $b^{\prime}=b$ and $\mathcal{A}$ has made neither $F_{I}$ Reveal nor $F_{M}$ Reveal query on any of the values $u_{1}, u_{2}$.

DEFINITION 2. We say that an ID-based concurrent signature scheme is ambiguous if no polynomially bounded adversary has non-negligibly advantage of winning in the above game.

Unlinkability. Unlinkability for a concurrent signature scheme is defined by the following game between an adversary $\mathcal{A}$ and a challenger $\mathcal{C}$.

- Setup: This is as above in the unforgeability game.

- Phase 1: $\mathcal{A}$ makes a sequence of EXTRACT, $F_{I}, F_{M}, F_{I}$ Reveal, $F_{M}$ Reveal, ASIGN queries. These queries are answered by $\mathcal{C}$ as in the above unforgeability game.

- Challenge:

- $\mathcal{A}$ selects a challenge tuple $\left(I D_{i}, I D_{j}, m_{i 0}, m_{i 1}, \sigma_{i 0}, \sigma_{i 1}\right)$ such that AVER$\operatorname{IFY}\left(\sigma_{i a}, I D_{i}, I D_{j}, m_{i a}\right)=$ accept for $a=0,1$, where $\sigma_{i a}=\left(u_{i a}, u_{j a}, V_{i a}\right)$.

- $\mathcal{C}$ randomly selects $b \in\{0,1\}, k_{j} \in \mathcal{K}_{M}$ and computes $f_{j}=F_{M}\left(k_{j}, u_{j b}\right)$.

- $\mathcal{C}$ selects $m_{j} \in \mathcal{M}$, outputs $\sigma_{j}=\left(u_{j}^{\prime}, u_{i}^{\prime}, V^{\prime}\right)=\operatorname{ASIGN}\left(I D_{j}, I D_{i}, S_{I D_{j}}, f_{j}, m_{j}\right)$.

- Phase 2: $\mathcal{A}$ may make another sequence of queries as in Phase 1; these are handled by $\mathcal{C}$ as before.

- Output: Finally $\mathcal{A}$ outputs a guess bit $b^{\prime} \in\{0,1\}$. $\mathcal{A}$ wins if $b^{\prime}=b$ and $\mathcal{A}$ has not made any $F_{M}$ Reveal query on $f_{j}$.

DEFINITION 3. We say that an ID-based concurrent signature scheme is unlinkable if no polynomially bounded adversary has non-negligibly advantage of winning in the above game.

Fairness. For the case of one keystone only, Chen et al. presented a formal definition of fairness in (Chen et al., 2004), but it is no longer appropriate in the case of two or more keystones. Chow et al. (Chow and Susilo, 2005) did not present any new definition of fairness, as a result, their schemes are unfair. Here we present a formal definition of fairness for the case of two keystones. Our definition uses the following game between an adversary $\mathcal{A}$ and a challenger $\mathcal{C}$ :

- Setup: This is as above in the unforgeability game.

- EXTRACT, $F_{I}, F_{M}, F_{I}$ Reveal, $F_{M}$ Reveal Queries: These queries are answered by $\mathcal{C}$ as in the above unforgeability game.

- ASIGN Queries: In the case of fairness, we have two ASIGN Queries as follows.

- IASIGN Query: $\mathcal{A}$ can request an ambiguous signature for any input of the form $\left(I D_{i}, I D_{j}, m_{i}\right)$ where $I D_{i}, I D_{j} \neq I D_{i}$ are the identities of the participants and $m_{i} \in \mathcal{M}$. $\mathcal{C}$ first gets a keystone-fix $f_{i}$ by a $F_{I}$ Query and then returns an ambiguous signature $\sigma=\left(u_{i}, u_{j}, V\right)$. 
- MASIGN Query: $\mathcal{A}$ can request an ambiguous signature for any input of the form $\left(I D_{i}, I D_{j}, m_{i}, f\right)$ where $I D_{i}, I D_{j} \neq I D_{i}$ are the identities of the participants, $m_{i} \in \mathcal{M}$ and $f \in \mathcal{F}$. $\mathcal{C}$ first gets a keystone-fix $f_{i}$ by a $F_{M}$ Query with input $f$ and then returns an ambiguous signature $\sigma=\left(u_{i}, u_{j}, V\right)$.

- Output: Finally, $\mathcal{A}$ chooses the challenge identities $I D_{c}$ and $I D_{d}$, outputs a signature $\sigma=(u, f, V)$ along with a message $m$.

The adversary wins the game if $\operatorname{AVERIFY}\left(\sigma, I D_{c}, I D_{d}, m\right)=$ accept and if either of the following cases hold:

- $\sigma$ is a previous output from a IASIGN Query. No $F_{I}$ Reveal query on $f$ was made and $\mathcal{A}$ produces a keystone $k$ such that $f=F_{I}(k)$ or produces a keystone pair $\left(k, k^{\prime}\right)$ such that $f=F_{M}\left(k, F_{I}\left(k^{\prime}\right)\right)$.

- $\sigma$ is a previous output from a MASIGN Query. $\mathcal{A}$ produces a keystone $k$ such that $f=F_{I}(k)$ or produces keystones $k_{1}, k_{2}$ and $k^{\prime}$ such that $f=$ $F_{M}\left(k_{1}, F_{I}\left(k_{2}\right)\right)=F_{M}\left(k^{\prime}, f^{\prime}\right)$ but $f^{\prime} \neq F_{I}\left(k_{2}\right)$, where $f^{\prime}$ is the keystone-fix in the input of MASIGN Query. In which $\mathcal{A}$ gets the signature $\sigma$.

DEFINITION 4. We say that an ID-based concurrent signature scheme is fair if a polynomially bounded adversary's probability of success in the above game is negligible.

DEFINITION 5. A ID-based concurrent signature scheme is secure if it is existentially unforgeable under a chosen message attack, correct, ambiguous, unlinkable and fair.

\section{Improved ID-Based Perfect Concurrent Signature Schemes}

In Chow et al.'s schemes, both keystones $k_{I}$ and $k_{M}$ are chosen by Alice, as a result, Alice can cheat Bob by carefully choosing the keystone fix $f_{I}$. If keystones $k_{I}$ and $k_{M}$ are chosen by Alice and Bob, respectively, all attacks above can be avoided. We present two simple but significant improvements as follows.

\subsection{Improved Scheme 1}

\subsubsection{Concurrent Signature Algorithms}

- SETUP:

- $\mathbb{G}_{1}, \mathbb{G}_{2}, \hat{e}, q, P, P_{\text {pub }}, H_{0}, H_{1}, \mathcal{M}, \mathcal{F}, \mathcal{K}^{\prime}$ are the same as that of the original scheme;

- sets $\mathcal{K}_{I}=\mathcal{K}_{M}=\mathbb{G}_{2}$;

- sets $F_{I}: \mathbb{G}_{2} \rightarrow \mathbb{Z}_{q}$ be a one-way permutation;

- sets $F_{M}(x, y)=F_{I}(x)+y \quad(\bmod q)$;

- sets $\operatorname{Enc}(k)=k P$;

- sets $\operatorname{Dec}\left(K^{\prime}, K^{\prime \prime}\right)=\hat{e}\left(K^{\prime}, K^{\prime \prime}\right)$;

- EXTRACT: The same as that of the original scheme. 
- ASIGN: The algorithm accepts $\left(I D_{i}, I D_{j}, S_{I D_{i}}, f_{i}, m_{i}\right)$ and performs the following:

- selects a random point $Z \in \mathbb{G}_{1}$;

- computes $u_{0}=H_{1}\left(H_{0}(m)\left\|\left(I D_{i} \oplus I D_{j}\right)\right\| \hat{e}(Z, P) \hat{e}\left(f_{i} Q_{I D_{j}}, P_{p u b}\right)\right)$;

- computes $V=u_{0}^{-1}\left(Z-\left(u_{0}-u_{j}\right) S_{I D_{i}}\right)$;

- sets $u_{i}=u_{0}-f_{i} \quad(\bmod q), u_{j}=f_{i}$;

- outputs $\sigma=\left(u_{i}, u_{j}, V\right)$ as the signature on message $m$.

- AVERIFY: The same as that of the original scheme.

- VERIFY: The algorithm accepts $\left(k_{i}, k_{j}, S^{\prime}\right)$, where $k_{i} \in \mathcal{K}_{I}$ and $k_{j} \in \mathcal{K}_{M}$ are the keystones and $S^{\prime}=\left(\sigma_{i}, \sigma_{j}, I D_{i}, I D_{j}, m_{i}, m_{j}\right)$. The algorithm verifies whether $f_{i}=F_{I}\left(k_{i}\right), f_{j}=F_{I}\left(k_{j}\right)+f_{i} \quad(\bmod q)$. If not, then outputs reject. Otherwise, run AVERIFY on $\sigma_{i}$ and $\sigma_{j}$ respectively. If both outputs are accept, then outputs accept. Otherwise, outputs reject.

\subsubsection{Concurrent Signature Protocol}

- Alice performs the following:

- picks a random keystone $k_{I} \in \mathbb{G}_{2}$, computes keystone fix $f_{I}=F_{I}\left(k_{I}\right)$;

- selects a message $m_{I} \in \mathcal{M}$, computes her ambiguous signature as $\sigma_{I}=$ $\left(u_{I}, u_{M}, V\right) \leftarrow \operatorname{ASIGN}\left(I D_{I}, I D_{M}, S_{I D_{I}}, f_{I}, m_{I}\right)$;

- sends $\sigma_{I}$ to Bob.

- Bob performs the following:

- verifies the signature $\sigma_{I}$ by testing whether AVERIFY $\left(\sigma_{I}, I D_{I}, I D_{M}, m_{I}\right)=a c$ cept. Aborts if the equation does not hold.

- picks a random number $k \in \mathbb{Z}_{q}$, computes keystone $k_{M}=\hat{e}\left(P_{p u b}, Q_{I D_{I}}\right)^{k}$;

- computes encrypted keystone $K_{M}=k P$;

- computes matching keystone fix $f_{M}=F_{I}\left(k_{M}\right)+u_{j} \quad(\bmod q)$;

- selects a message $m_{M} \in \mathcal{M}$, and computes his ambiguous signature as $\sigma_{M}=$ $\left(u_{M}^{\prime}, u_{I}^{\prime}, V^{\prime}\right) \leftarrow \operatorname{ASIGN}\left(I D_{M}, I D_{I}, S_{I D_{M}}, f_{M}, m_{M}\right) ;$

- sends $\sigma_{M}$ and $K_{M}$ to Alice.

- Alice verifies $\sigma_{M}$ by testing whether

$-u_{I}^{\prime}=F_{I}\left(\hat{e}\left(K_{M}, S_{I D_{I}}\right)\right)+u_{M} \quad(\bmod q)$;

- $\operatorname{AVERIFY}\left(\sigma_{M}, I D_{M}, I D_{I}, m_{M}\right)=$ accept.

If not, then Alice aborts. Otherwise, Alice computes keystone $k_{M}=\hat{e}\left(K_{M}, S_{I D_{I}}\right)$ and releases the keystone $\left(k_{I}, k_{M}\right)$, then both signatures are binding concurrently.

\subsection{The Security}

The correctness of the improved concurrent signature scheme 1 can easily be verified.

Since we do not make any change to the ASIGN algorithm, the unforgeability of our improved scheme is kept the same as the original scheme. So, the same as that of (Chow and Susilo, 2005), we have following lemma. Its proof follows the proof of the Lemma 1 in (Chen et al., 2004) given by Chen et al. Due to space limitation, the proof is omitted. 
Lemma 1 (unforgeability). The improved concurrent signature scheme 1 is existentially unforgeable under a chosen message attack in the random oracle model, assuming the hardness of Co-CDH problem.

Lemma 2 (ambiguity). The improved concurrent signature scheme 1 is ambiguous in the random oracle model.

We consider the following distributions:

$$
\xi=\left\{\begin{array}{l|l}
\left(u_{1}, u_{2}, V\right) & \begin{array}{l}
Z \in \in_{R} \mathbb{G}_{1}, \quad k \in_{R} \mathbb{G}_{2} \\
u_{2}=F_{I}(k) \\
u_{0}=H_{1}\left(H_{0}(m)\left\|\left(I D_{i} \oplus I D_{j}\right)\right\| \hat{e}(Z, P) \hat{e}\left(u_{2} Q_{I D_{j}}, P_{p u b}\right)\right) \\
u_{1}=u_{0}-u_{2} \\
V=u_{0}^{-1}\left(Z-u_{1} S_{I D_{i}}\right)
\end{array}
\end{array}\right\}
$$

and

$$
\zeta=\left\{\begin{array}{l|l}
\left(u_{2}^{\prime}, u_{1}^{\prime}, V^{\prime}\right) & \begin{array}{l}
Z \in_{R} \mathbb{G}_{1}, \quad k \in_{R} \mathbb{G}_{2} \\
u_{2}^{\prime}=F_{I}(k) \\
u_{0}^{\prime}=H_{1}\left(H_{0}(m)\left\|\left(I D_{i} \oplus I D_{j}\right)\right\| \hat{e}(Z, P) \hat{e}\left(u_{2}^{\prime} Q_{I D_{i}}, P_{p u b}\right)\right) \\
u_{1}^{\prime}=u_{0}^{\prime}-u_{2}^{\prime} \\
V^{\prime}=u_{0}^{\prime-1}\left(Z-u_{1}^{\prime} S_{I D_{j}}\right)
\end{array}
\end{array}\right\}
$$

In the random oracle model, the distributions of the outputs of $F_{I}$ and $H_{1}$ are uniform, so two distributions above are the same.

The distribution of $F_{I}(k)+F_{I}\left(k^{\prime}\right)$ is the same as the distribution of $F_{I}(k)$, so the case of $f=F_{I}(k)+F_{I}\left(k^{\prime}\right)$ is the same as that of $f=F_{I}(k)$.

Hence, the adversary wins the game of Definition 3 with probability exactly $1 / 2$, so the scheme is ambiguous.

Lemma 3 (Unlinkability). The improved concurrent signature scheme 1 is unlinkable. Since $F_{I}: \mathbb{G}_{2} \rightarrow \mathbb{Z}_{q}$ is a one-way permutation, given $f_{j}, f_{i 0}, f_{i 1}$, there exist $k_{0}$ and $k_{1}$ such that $f_{j}=F_{I}\left(k_{0}\right)+f_{i 0}$ and $f_{j}=F_{I}\left(k_{1}\right)+f_{i 1}$, respectively. Such $k_{0}$ and $k_{1}$ always exist regardless of the values of $f_{j}$ and $\left(f_{i 0}, f_{i 1}\right)$, so $f_{j}$ and $f_{i b}$ have exactly the same relation defined by $F_{M},(b=0,1)$. Therefore, even an infinitely powerful adversary wins the game of Definition 4 with probability exactly $1 / 2$, so the scheme is unconditional unlinkable.

Lemma 4 (fairness). The improved concurrent signature scheme 1 is fair in the random oracle model.

Suppose that there exists an algorithm $\mathcal{A}$ that with probability $\delta$ wins the game in Definition 5, we show that $\delta$ is negligible. Let $\mu_{I}$ and $\mu_{M}$ are the numbers of $F_{I}$ and 
$F_{M}$ queries made by $\mathcal{A}$ and let $\mu_{I S}$ and $\mu_{M S}$ are the numbers of IASIGN and MASIGN queries made by $\mathcal{A}$, respectively.

If case 1 of the output conditions occurs, then $\sigma$ is a previous output from a IASIGN Query and $f$ is a previous output of a $F_{I}$ query. We discuss the following two cases.

- $\mathcal{A}$ has found a keystone $k$ such that $f=F_{I}(k)$ but without making any $F_{I}$ Reveal query on input $f$. In the random oracle model, $\mathcal{A}$ 's probability of producing such a $k_{c}$ is at most $\mu_{I S} \mu_{I} / q$.

- $\mathcal{A}$ has found a keystone pair $\left(k, k^{\prime}\right)$ such that $f=F_{I}(k)+F_{I}\left(k^{\prime}\right)$. In the random oracle model, $\mathcal{A}$ can get such $\left(k, k^{\prime}\right)$ by $F_{M}$ query with probability at most $\mu_{I S} \mu_{M} / q$ or by $F_{I}$ query with probability at most $\mu_{I S} \mu_{I}\left(\mu_{I}+1\right) / 2 q$, where $\mu_{I}\left(\mu_{I}+1\right) / 2 q$ is the probability of two outputs of $F_{I}$ queries $f^{\prime}$ and $f^{\prime \prime}$ such that $f=f^{\prime}+f^{\prime \prime}$. So, $\mathcal{A}$ 's probability of producing such $\left(k, k^{\prime}\right)$ is at most $\mu_{I S}\left(\mu_{I}\left(\mu_{I}+1\right)+2 \mu_{M}\right) / 2 q$.

If case 2 of the output conditions occurs, then $\sigma$ is a previous output from a MASIGN Query. In this case, $f$ is a previous output of a $F_{M}$ query on input of some $f^{\prime}$, so $\mathcal{A}$ can get $k^{\prime}$ and $f^{\prime}$ such that $f=F_{I}\left(k^{\prime}\right)+f^{\prime}$ by a $F_{M}$ Reveal query. We would like to emphasize the fact that. In our scheme, the $f^{\prime}$ was set before choosing the $k^{\prime}$, which is the significant difference between our scheme and that of (Chow and Susilo, 2005). Thus $\mathcal{A}$ can neither find the required keystone $k$ by setting $f^{\prime}=F_{I}(k)-F_{I}\left(k^{\prime}\right)$, nor find the required keystones $k_{1}$ and $k_{2}$ by setting $f^{\prime}=F_{I}\left(k_{1}\right)+F_{I}\left(k_{2}\right)-F_{I}\left(k^{\prime}\right)$. So the case 2 was reduced to the case 1 .

Since $\mu_{I}, \mu_{M}, \mu_{I S}$ and $\mu_{M S}$ are polynomially bounded in the security parameter $\left\lceil\log _{2} q\right\rceil$, the probability $\delta$ is negligible.

Theorem 1. The improved concurrent signature scheme 1 is secure in the random oracle model, assuming the hardness of $\mathrm{Co}-\mathrm{CDH}$ problem.

The proof follows directly from above lemmas.

\subsection{Improved Scheme 2}

Chow et al.'s Scheme 2 can be improved in the similar way as following.

\subsubsection{Concurrent Signature Algorithms}

- SETUP:

- $\mathbb{G}_{1}, \mathbb{G}_{2}, \hat{e}, q, P, P_{\text {pub }}, H_{0}, H_{1}, \mathcal{M}, \mathcal{F}, \mathcal{K}^{\prime}$ are the same as that of the original scheme;

- sets $\mathcal{K}_{I}=\mathcal{K}_{M}=\mathbb{G}_{2}$

- sets $F_{I}: \mathbb{G}_{2} \rightarrow \mathbb{G}_{1}$ be a one-way permutation;

- sets $F_{M}(x, y)=F_{I}(x)+y$;

- sets $\operatorname{Enc}(k)=k P$;

- sets $\operatorname{Dec}\left(K^{\prime}, K^{\prime \prime}\right)=\hat{e}\left(K^{\prime}, K^{\prime \prime}\right)$.

- EXTRACT: The same as that of the original scheme.

- ASIGN: The algorithm accepts $\left(I D_{i}, I D_{j}, S_{I D_{i}}, f_{i}, m_{i}\right)$ and performs the following: 
- sets $U_{j}=f_{i}$, computes $h_{j}=H_{1}\left(m\left\|\left(I D_{i} \oplus I D_{j}\right)\right\| U_{j}\right)$;

- chooses $r_{i}^{\prime} \in \mathbb{Z}_{q}^{*}$ randomly, computes $U_{i}=r_{i}^{\prime} Q_{I D_{i}}-U_{j}-h_{j} Q_{I D_{j}}$;

- computes $h_{i}=H_{1}\left(m\left\|\left(I D_{i} \oplus I D_{j}\right)\right\| U_{i}\right)$ and $V=\left(h_{i}+r_{i}^{\prime}\right) S_{I D_{i}}$;

- outputs the signature $\sigma=\left(U_{i}, U_{j}, V\right)$.

- AVERIFY: The same as that of the original scheme.

- VERIFY: The same as that of the improved scheme 1.

\subsubsection{Concurrent Signature Protocol}

- Alice performs the following:

- picks a random keystone $k_{I} \in \mathcal{K}_{I}$, computes keystone fix $f_{I}=F_{I}\left(k_{I}\right)$;

- selects a message $m_{I} \in \mathcal{M}$, computes her ambiguous signature as $\sigma_{I}=$ $\left(U_{I}, U_{M}, V\right) \leftarrow \operatorname{ASIGN}\left(I D_{I}, I D_{M}, S_{I D_{I}}, f_{I}, m_{I}\right) ;$

- sends $\sigma_{I}$ to Bob.

- Bob performs the following:

- verifies the signature $\sigma_{I}$ by testing whether $\operatorname{AVERIFY}\left(\sigma_{I}, I D_{I}, I D_{M}, m_{I}\right) \stackrel{?}{=} a c$ cept holds. Aborts if the equation does not hold.

- picks a random number $k \in \mathbb{Z}_{q}$;

- computes keystone $k_{M}=\hat{e}\left(P_{p u b}, Q_{I D_{I}}\right)^{k}$;

- computes encrypted keystone $K_{M}=k P$, computes matching keystone fix $f_{M}=F_{I}\left(k_{M}\right)+U_{M}$

- selects a message $m_{M} \in \mathcal{M}$, and computes his ambiguous signature as $\sigma_{M}=$ $\left(U_{M}^{\prime}, U_{I}^{\prime}, V^{\prime}\right) \leftarrow \operatorname{ASIGN}\left(I D_{M}, I D_{I}, S_{I D_{M}}, f_{M}, m_{M}\right) ;$

- sends $\sigma_{M}$ and $K_{M}$ to Alice.

- Alice verifies $\sigma_{M}$ by testing whether

- $U_{I}^{\prime}=F_{I}\left(\hat{e}\left(K_{M}, S_{I D_{I}}\right)+U_{M}\right.$

- $\operatorname{AVERIFY}\left(\sigma_{M}, I D_{M}, I D_{I}, m_{M}\right)=$ accept.

If not, then Alice aborts. Otherwise, Alice computes keystone $k_{M}=\hat{e}\left(K_{M}, S_{I D_{I}}\right)$ and releases the keystone $\left(k_{I}, k_{M}\right)$, then both signatures are binding concurrently.

Similar to the improved scheme 1, we have the following theorem.

Theorem 2. The improved concurrent signature scheme 2 is secure in the random oracle model, assuming the hardness of Co-CDH problem.

\section{Conclusion}

Concurrent signatures were introduced as an alternative approach to solving the problem of fair exchange of signatures and several concrete concurrent signature schemes have been proposed. In this paper, we present attacks on the fairness of Chowet et al.'s identitybased perfect concurrent signature schemes (Chow and Susilo, 2005). We also present a modified definition of ID-based concurrent signatures which redress the flaw of Chow et al.'s definition and propose two improved schemes to fix our attacks. 


\section{References}

Asokan N., V. Shoup and M. Waidner (1998). Optimistic fair exchange of signatures. In Advances in Cryptology - EUROCRYPT '98, Lecture Notes in Computer Science, vol. 1403. Springer-Verlag, Berlin, pp. 591-606.

Boneh, D., C. Gentry, B. Lynn and H. Shacham (2003). Aggregrate and verifiably encrypted signatures from bilinear maps. In Advances in Cryptology - EUROCRYPT 2003, Lecture Notes in Computer Science, vol. 2656. Springer-Verlag, Berlin, pp. 416-432.

Boneh, D., and M. Naor (2000). Timed commitments (extended abstract). In Advances in Cryptology - CRYPTO 2000, Lecture Notes in Computer Science, vol. 1880. Springer-Verlag, Berlin, pp. 236-254.

Camenisch, J., and V. Shoup (2003). Practical verifiable encryption and decryption of discrete logarithms. In Advances in Cryptology - CRYPTO 2003, Lecture Notes in Computer Science, vol. 2729. Springer-Verlag, Berlin, pp. 126-144.

Chen, L., C. Kudla and K. G. Paterson (2004). Concurrent signatures. In Advances in Cryptology - EUROCRYPT 2004, Lecture Notes in Computer Science, vol. 3027. Springer-Verlag, Berlin, pp. 287-305.

Chow, S., and W. Susilo (2005). Generic construction of (identity-based) perfect concurrent signatures. In Information and Communications Security (ICICS 2005), Lecture Notes in Computer Science, vol. 3783. Springer-Verlag, Berlin, pp. 194-206.

Even, S., O. Goldreich and A. Lempel (1985). A randomized protocol for signing contracts. Commun. ACM, 28(6), 637-647.

Gangishetti, R., M. Gorantla, M. Das, A. Saxena (2006). Identity based multisignatures. Informatica, 17(2), $177-186$

Garay, J., M. Jakobsson and P. MacKenzie (1999). Abuse-free optimistic contract signing. In Advances in Cryptology - CRYPTO 1999, Lecture Notes in Computer Science, vol. 1666. Springer-Verlag, Berlin, pp. 449466.

Garay, J., and C. Pomerance (2003). Timed fair exchange of standard signatures. In Proc. Financial Cryptography 2003, Lecture Notes in Computer Science, vol. 2742. Springer-Verlag, Berlin, pp. 190-207.

Goldreich, O. (1983). A simple protocol for signing contracts. In Advances in Cryptology - CRYPTO '83. Springer-Verlag, Berlin, pp. 133-136.

Kancharla, P., S. Gummadidala, A. Saxena (2007). Identity based strong designated verifier signature scheme. Informatica, 18(2), 239-252.

Nguyen, K. (2005). Asymmetric concurrent signatures Khanh Nguyen. In Information and Communications Security (ICICS 2005), Lecture Notes in Computer Science, vol. 3783. Springer-Verlag, Berlin, pp. 181193.

Shamir, A. (1985). Identity-base cryptosystems and signature schemes. In Advances in Cryptology - Crypto\&84, Lecture Notes in Computer Science, Vol. 196. Springer-Verlag, Berlin, pp. 47-53.

Susilo, W., and Y. Mu (2005). Tripartite concurrent signatures. In The 20th IFIP International Information Security Conference (IFIP/SEC 2005), Springer, pp. 425-441.

Susilo, W., Y. Mu and F. Zhang (2004). Perfect concurrent signature schemes. In ICICS 2004, Lecture Notes in Computer Science, vol. 3269. Springer-Verlag, Berlin, pp. 14-26.

Tonien, D., W. Susilo and R. Safavi-Naini (2006). Multi-party concurrent signatures. In ISC 2006, Lecture Notes in Computer Science, vol. 4176. Springer-Verlag, Berlin, pp. 131-145. 
Z. Huang obtained the PhD degree in cryptology from School of Communication Engineering at Xidian University in 2005. Now he is a professor and the director of Department of Computer Science and Engineering at Zhangzhou Normal University. His current research interests focus on the areas of cryptology, electronic commerce security, and network security etc.

K. Chen obtained the BS and the MS degrees in applied mathematics from Xidian University in 1982 and 1985, respectively, and the PhD degree from Justus-Liebig University, Germany, in 1994. Now he is a professor and a doctoral supervisor of Department of Computer Science and Engineering, a vice dean of School of Information Security Engineering, the director of Cryptography and Information Security Lab at Shanghai Jiao Tong University. His current research interests focus on the areas of cryptography, information security and coding theory etc.

X. Lin is an associate professor of Department of Computer Science and Engineering at Zhangzhou Normal University. His current research interests focus on the areas of cryptology and information security.

R. Huang is an associate professor of Department of Computer Science and Engineering at Zhangzhou Normal University. Her current research interests focus on the areas of cryptology and network security. 


\section{Dvieju tapatybe pagrịstu tobulų konkuruojančiu parašu schemu analizè ir pagerinimai}

Zhenjie HUANG, Kefei CHEN, Xuanzhi LIN, Rufen HUANG

Konkuruojančiu parašų sąvoka buvo pristatyta Chen, Kudla ir Paterson Eurocrypt2004 straipsnyje. Konkuruojančiu parašu schemoje dvi esybès gali sugeneruoti du parašus, kurie yra nesusieti, kol papildoma informacija (kertinis akmuo) nèra paskelbiama vienos iš šalių. Paskelbus kertini akmeni abu parašai tampa konkuruojančiai susieti su tikraisiais pasirašančiaisiais. ICICS2005 konferencijoje Chow ir Susilo pasiūle dvi tapatybe pagristas tobulas konkuruojančiu parašu schemas. Šiame straipsnyje mes parodome, kad šios dvi schemos yra neteisingos. Jose pirminis pasirašantysis gali apgaudinèti antrinị pasirašantiji . Mes pristatome formalu ID paremtą konkuruojančiu parašu apibrežimą ir tada pasiūlome du paprastus bet svarbius pagerinimus mūsų ataku sutvarkymui. 